\title{
Anisometropic and strabismic amblyopia in the age group 2 years and above: a prospective study of the results of treatment
}

\author{
Joan Lithander, Johan Sjöstrand
}

\begin{abstract}
Forty-four children aged 2-9 years with strabismic and anisometropic amblyopia were prospectively followed up during amblyopia treatment. The efficacy of optimised treatment in terms of number of cured children, time to achieve cure, and rate of initial improvement of visual acuity was evaluated in relation to age at start of treatment, type and initial degree of amblyopia, and adherence to treatment regimen. Compliance with treatment was the most critical factor predicting a successful outcome. Among the compliant children 35 out of 36 were cured (visual acuity difference between amblyopic and non-amblyopic eyes not more than one line) within five months regardless of age, treatment regimen, and type or initial degree of amblyopia as compared with none in the group with low compliance. Most of these compliant children were cured within three months, with shorter treatment times on average for the younger children. The initial improvement of visual acuity was also faster at 2 years than at 4 years of age. Anisometropes with moderate amblyopia at the start of treatment were over-represented in the group with low compliance. We conclude that early diagnosis of strabismus in combination with general population screening at the age of 4 to detect amblyopia caused by anisometropia or microstrabismus seems to be efficacious for the cure of most cases. The major factor in treatment failure was found to be inadequate adherence to the treatment regimen.
\end{abstract}

At 4 years of age the Swedish population is screened for subnormal vision. One major aim is to detect amblyopia by measuring visual acuity at the child health centres. Nordlöw and Joachimsson ${ }^{1}$ and Köhler and Stigmar ${ }^{2}{ }^{3}$ initiated this programme and found it efficacious.

The questions we asked were: Could anything be gained by lowering the screening age? Is earlier treatment more effective and associated with a better outcome? Few systematic studies have evaluated the effect of factors such as age below 4 and type and degree of amblyopia in the treatment of the clinically most common forms of amblyopia, that is, those caused by strabismus or anisometropia. ${ }^{45}$

Most clinicians agree that treatment of amblyopia is less favourable if it appears during the first months of life and if it is associated with extreme degrees of anisometropia. ${ }^{6}$ But the relative importance of various factors for its successful treatment in the more usual clinical situation is not well defined. The efficacy of screening for the treatment of amblyopia has also been questioned during recent years. ${ }^{7-9}$

The recent development of accurate methods for testing visual acuity with optotypes in younger children down to an approximate age of 2 years $^{10}$ (Lithander, in preparation) has enabled us to study the efficacy of amblyopia treatment as related to optotype acuity over an extended time period. At the same time more interest has been focused on the psychological implications of amblyopia treatment in an active child, and low compliance has been considered to be a major reason for treatment failure in older children. ${ }^{5}$

In this prospective clinical study of children aged 2 years or more we have evaluated the association of various factors such as age at start of treatment and initial degree or type of amblyopia with efficacy of amblyopia treatment. Furthermore, we have analysed factors of importance for the successful outcome of amblyopia therapy.

\section{Patients and methods}

\section{PATIENTS}

Forty four children aged 2 to 9 years with unilateral amblyopia diagnosed by the first author during the time period 1983-7 at the Paediatric Ophthalmology Unit of Östra Hospital, Göteborg, and fulfilling the inclusion criteria were enrolled consecutively in the study. Inclusion criteria were previously untreated unilateral amblyopia due to strabismus $(n=27)$, or anisometropia without strabismus $(n=17)$, persisting after eight or more weeks of spectacle wear if optical correction was needed. All patients with large angle strabismus, microstrabismus, or strabismus in combination with anisometropia were classified as strabismus. Amblyopia was defined as a difference of at least two lines on the acuity chart between the eyes when tested with best correction after eight weeks with spectacles when needed.

They were mainly referred from the child health centres of Göteborg at any age below 7 years either for squinting problems or for subnormal vision detected at the visual acuity screening test at the age of 4 . There were 22 boys and 22 girls. All the children were followed up for at least three years following the start of treatment (mean follow-up time 4.5 years).

CLINICAL EXAMINATION

After retinoscopy optical correction was prescribed when needed for full time use. Aniso- 


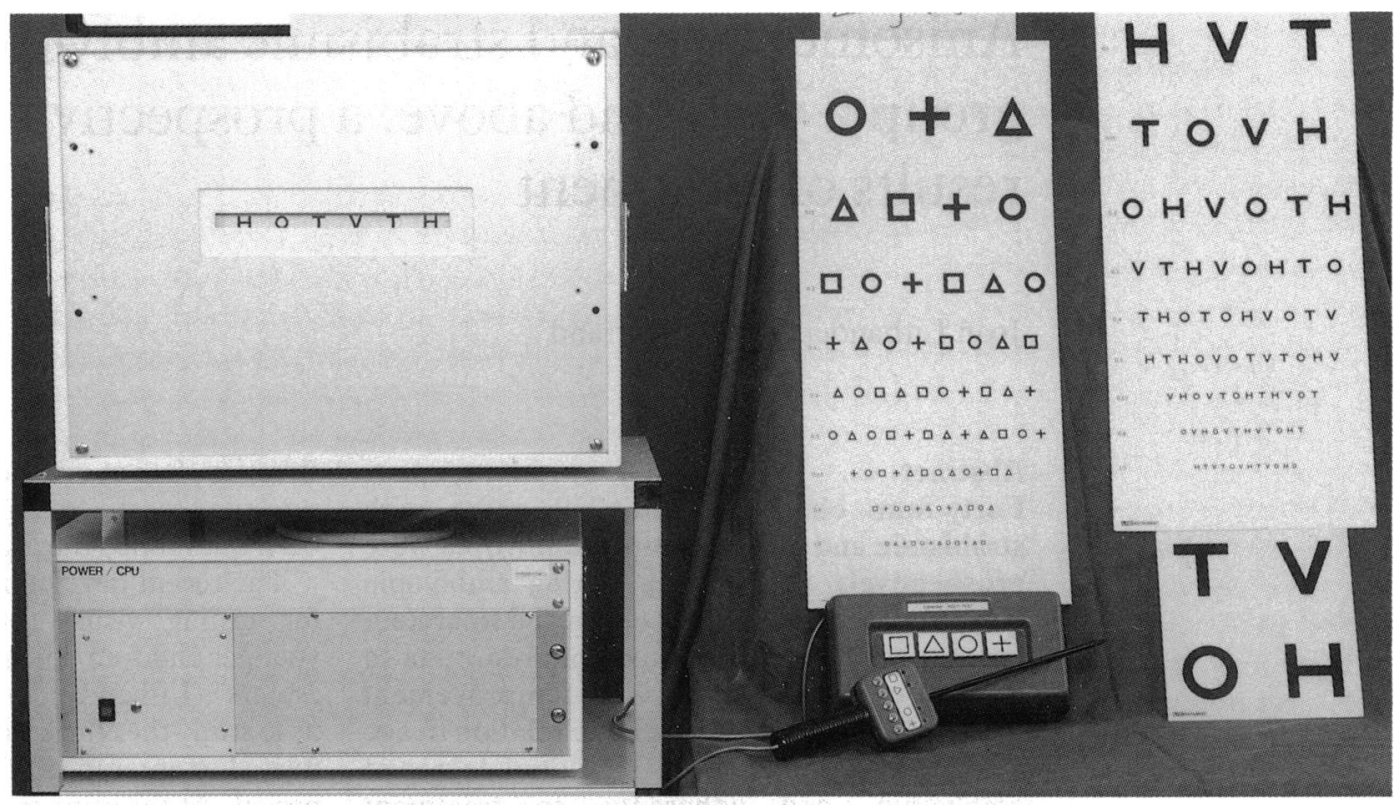

Figure 1 Design principles of the $\mathrm{O}$ test, the Kolt test, and HVOT test.

metropia was defined as the difference of one dioptre or more in any symmetrical meridian. The clinical characteristics of the children are summarised in Table 1.

Visual acuity (VA) was assessed at the age 2 to $2 \frac{1}{2}$ years with the O-test (Lithander, in preparation). The $\mathrm{O}$-test is a new method designed for testing visual at the age of 18 months and above. It has a testing distance of $40 \mathrm{~cm}$. The child has to point out the $\mathrm{O}$ among six symbols, for instance HVTOVH. The $\mathrm{O}$ is shown in different positions. It tests visual acuity for single symbols as the interspace between the letters is large. As soon as the child understood the matching symbol principle, the Kolt test with four alternative optotypes (triangle, square, circle, and cross) was used ${ }^{10}$ (Lithander, in preparation). The testing distance is $3 \mathrm{~m}$. The children have a matching box with the symbols on four buttons. They have to press the button corresponding to the optotype indicated at the test chart. As reinforcement a melody is played if they perform correctly. The $\mathrm{O}$ test, the Kolt test, and the HVOT test are shown in Figure 1. At the age of 4 the HVOT' was the elected test. For all the tests above the acuity was tested in approximately logarithmic steps from 0.05 or 0.1 to 1.0 . The visual acuity was defined as the line at which $3 / 4$ of the optotypes were correctly identified. In cases with VA below $0 \cdot 1$ the testing distance was decreased appropriately and the values transformed.

After the age of 5 visual acuity was tested with Snellen's E chart, and at near Haase's C test with 2.6 arc minutes of interspace between the optotypes. ${ }^{12}$ Snellen's letter chart was used, in general, after 7 years of age.

\section{TREATMENT}

The primary treatment in all cases was patching on the skin in front of the good eye. The time period for repeated occlusion was selected according to age. Thus a 2-year-old was treated with two days of occlusion and one free day; a 5year-old child was patched five days and had one free. Sixteen out of 27 children with strabismus (63\%) and 10 out of 17 anisometropes (59\%) had no other treatment (except glasses) than patching on the skin until cure. If the child refused or was unable to wear a patch on the skin the second choice of treatment was instillation of atropine in the best eye, if the acuity of the amblyopic eye was above $0 \cdot 2$. In these cases $0.5 \%$ atropine was instilled daily for one week followed by one week without treatment, and the treatment cycle was repeated until cure. When the residual amblyopia was mild and problem with the patching occurred, another choice of treatment was a Ryser filter attached to the glass of the good eye.

In order to monitor rate of improvement, time until first cure, and to optimise compliance the children were examined so far as possible every week during the first four weeks and then every second week for the following four weeks. After the start of the third month of follow-up examinations were carried out once a month until cure - that is when one line VA difference or less between the two eyes was achieved. After that, treatment was continued on a part time basis (occlusion of the good eye for one or two hours daily), preferably at children's TV time, until the acuity was equal in both eyes. An exception was in cases of microstrabismus. In this group the endpoint for treatment was one line of acuity difference between the eyes in order to avoid diplopia.

\section{COMPLIANCE}

A great effort was made to obtain optimal compliance with treatment. Any problems with either glasses, patching, atropine, Ryser filter, or follow-up were recorded. Low compliance was noted if the prescribed glasses were not worn, if appointments were missed more than once, and if atropine or the Ryser filter was not properly used after a period with problems with patching. Compliance was carefully controlled, as we met the family weekly at the beginning. Detailed information and instructions to the parents and the children before and during treatment were 
Table 1 Clinical characteristics: grade of amblyopia and compliance $(c)$

\begin{tabular}{lllll}
\hline \multicolumn{4}{c}{ Number of cases } \\
\hline $\begin{array}{lllll}\text { Grade of amblyopia } \\
\text { (VA at start) }\end{array}$ & Anisometropia & \multicolumn{2}{l}{ Strabimus } \\
\cline { 2 - 3 } \cline { 5 - 5 } & good c & low c & good c & low c \\
\hline$<0 \cdot 1$ & - & - & 6 & 1 \\
$0 \cdot 1-0.3$ & 3 & 5 & 15 & 2 \\
$0 \cdot 4-0.8$ & 9 & - & 3 & - \\
\cline { 2 - 5 } Total & 12 & 5 & 24 & 3 \\
\hline
\end{tabular}

regarded as essential. The same ophthalmologist (JL) and nurse were responsible for the examinations and for giving information to the parents.

\section{Results}

The adherence of the 44 children to the amblyopia therapy was graded. Thirty six of the children had good compliance to any form of amblyopic treatment (82\%) whereas eight children had low compliance (Table 1).

Of the 36 children with good compliance to any form of treatment 35 were cured according to our definition (one line of acuity difference between the eyes) regardless of type of amblyopia or grade of initial amblyopia in the age group 2 to 7 years. The only 'failure' in this group was a 6year-old girl with a high degree of anisometropia (retinoscopy right eye $+2 \cdot 5 \mathrm{sph}$, left eye $-6 \cdot 0$ sph -cyl 4.0 ax $\mathrm{O}^{\circ}$ ) combined with a monolateral esotropia. She improved from 0.05 to 0.4 in 90 days, after which no further improvement was possible in spite of optimal compliance. She was the only child who had contact lenses instead of glasses.

The duration of treatment until cure in relation to the age at the start of treatment of compliant children is presented in Figure 2. The treatment time needed to achieve cure for the strabismic children was related to age, with shorter time periods needed on average for the younger

Figure 2 Time to cure (days) vs age (years) for all cured compliant cases with strabismus $(n=23)$ and anisometropia $(n=12)$. Strabismus $\square$. Anisometropia $\bigcirc$

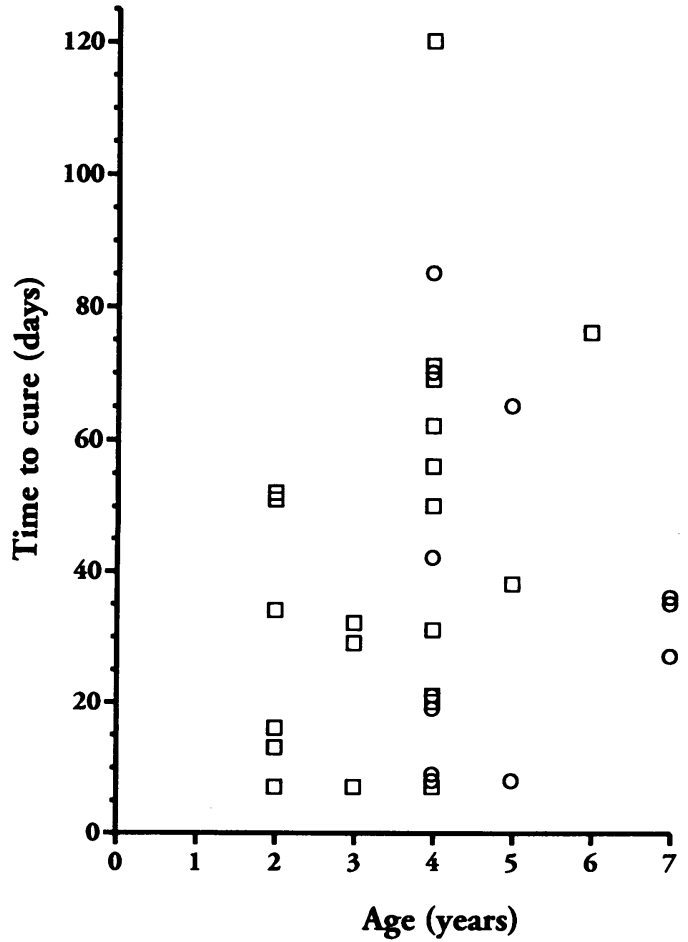

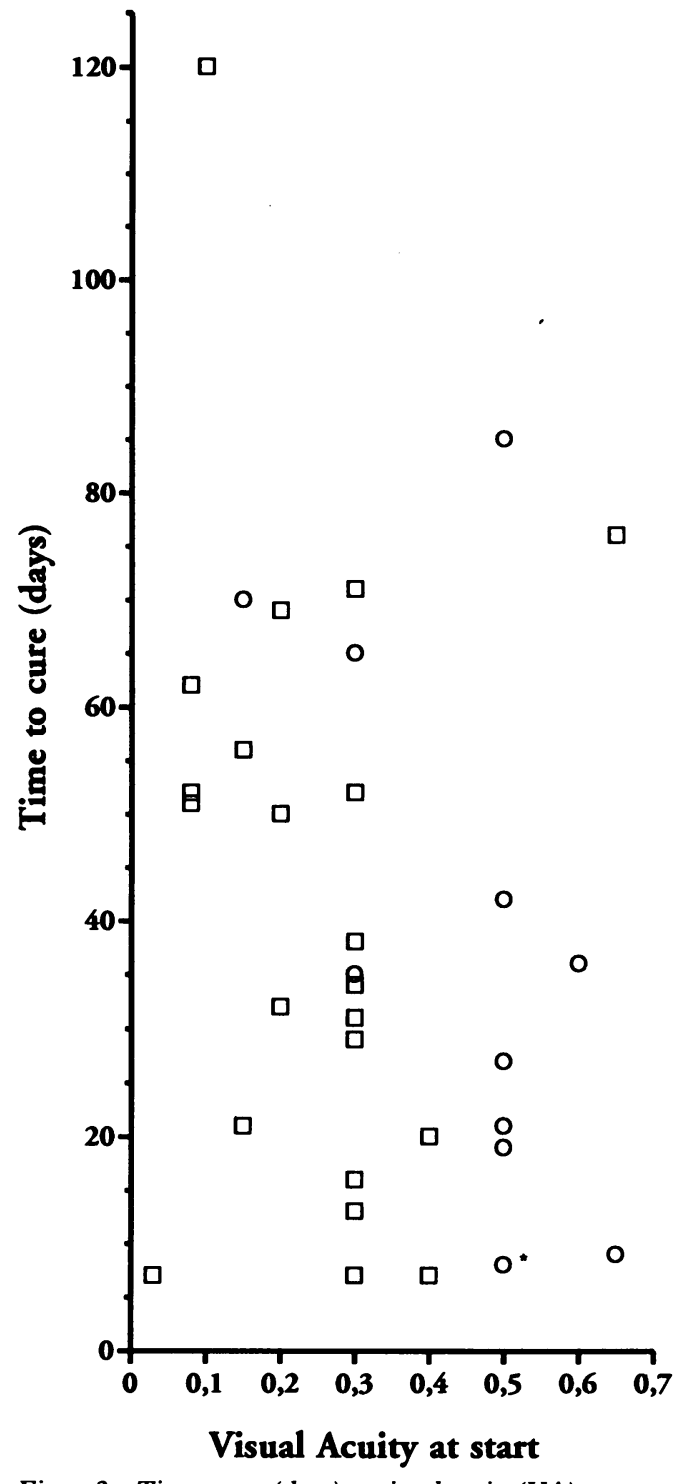

Figure 3 Time to cure (days) vs visual acuity (VA) at start of therapy for all cured compliant children $(n=35)$. Indicates two identical data points. Strabismus $\square$. Anisometropia $\bigcirc$.

children. For the compliant anisometropes no obvious relation to age was found in the limited age range studied for this group. A more direct comparison between the treatment time needed for stabismic $(n=8)$ or anisometropic $(n=7)$ children aged 4 years, all treated only with repeated patching, revealed that time to cure was in a similar range for both groups in spite of a generally lower VA at the start for the strabismic group. The treatment time until cure ranged from 10 to 80 days for all 4-year-old children compliant to patching. The treatment time to cure for most strabismic and anisometropic cases between 2 and 7 years old with good compliance to any form of amblyopic therapy was 100 days or less (Fig 2). It was not related to initial degree of amblyopia (Fig 3)

Although the treatment regimens other than patching were successful in cases with good compliance, the treatment time to cure, in general, was longer than that for the group with patching. One 7-year-old boy (case 4, outside the time scale in Figs 2 and 3) with microstrabismus (initial VA of $0 \cdot 2$ ) and treatment with atropine had a treatment time (over 200 days) strikingly longer than the others. However, six children 
out of 10 with atropine or Ryser filter treatment were cured within 50 days or less.

To compare the rate of improvement with respect to age and type or grade of amblyopia we evaluated the time needed to improve to the logarithmic half point of VA improvement between start and cure (Figs 4A, B). The time needed to reach this half point was on average shorter at 2 years than at 4 years of age for the strabismic children (Fig 4A). No obvious correlation was found between VA at start of treatment and the time to improve to this half point (Fig 4B). The average improvement during the first weeks of treatment in relation to VA at the start is demonstrated in Fig 5 for three representative groups children, namely those with visual acuity at the start of $\leqslant 0 \cdot 1,0 \cdot 3$, and 0.5 .

Of the total group of 44 children eight showed no or low compliance during the whole or part of the treatment period (Tables 1 and 2). Four of the children in this group were cured in spite of low compliance, but a long treatment period was needed (156 to 412 days). Among the compliant children achieving cure $(n=35)$ only one had a treatment time in this time range. Five of the cases with low compliance were anisometropes without strabismus with moderate amblyopia (VA $0 \cdot 1-0 \cdot 3$ ) at start of treatment (Table 1). Among all the anisometropic cases with moderate amblyopia at the start the non-compliant children accounted for almost $2 / 3$ (Table 1 ). The mean age of the children with low compliance was $5 \cdot 1$ years and that of children with good compliance $4 \cdot 2$ years at the start of treatment. However, an age of 2 years (case 18 and 38 ) was no guarantee of good compliance.

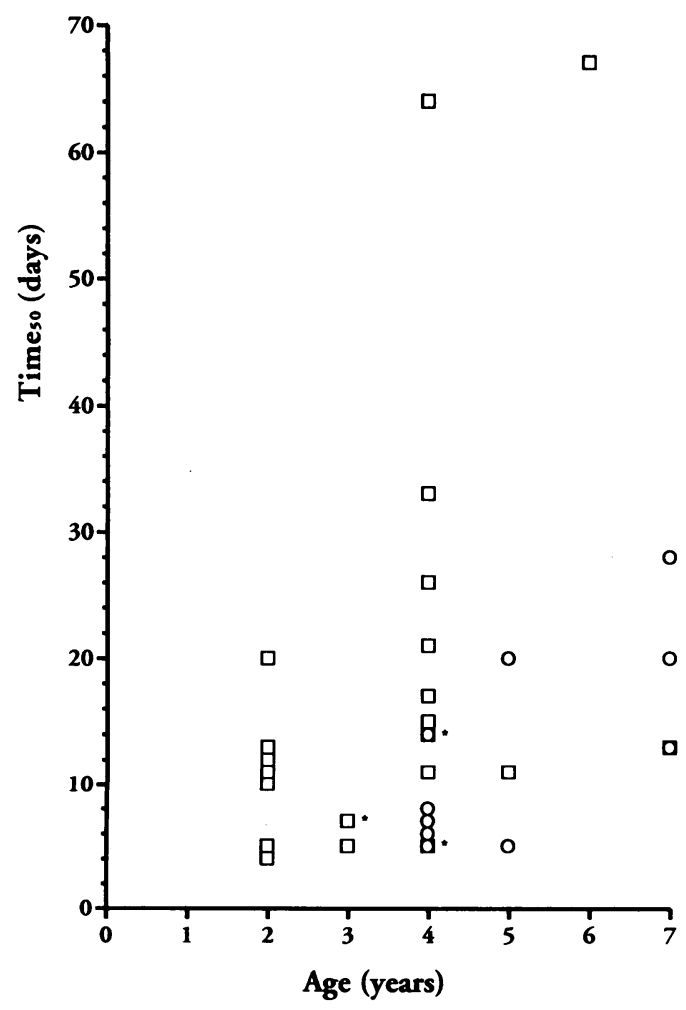

Figure 4A
Occlusion amblyopia was not registered in our 36 compliant children during the treatment period described here, though we observed that three cases on one occasion had one line of poorer performance in the good eye. At the following test the visual acuity returned to normal, though occlusion of the good eye was continued.

\section{Discussion}

This prospective study of children with previously untreated amblyopia was initiated in order to delineate limiting factors for cure and to find the reason for failures in different age groups from 2 years and above. A prerequisite for the study of age as a predicting factor for the outcome of amblyopia treatment is tests of acuity suitable for ages from 2 years. In all children below 3 years of age included in this study we could get reliable and reproducible information on visual acuity based on the same general test principle as those used for the older children ${ }^{1013}$ (Lithander in preparation). The main difference among the optotype tests was the inability to test crowding at the youngest age, since the $\mathrm{O}$ test tests acuity for single symbols at near (Lithander, in preparation).

The main question in our study was whether anything could be gained by lowering the age for screening of amblyopia below 4 years. We found no crucial age related differences in the final outcome of treatment, even though the younger age groups showed shorter treatment times. All but one of the compliant children were cured, regardless of age (between 2 and 7 years), diagnosis, and severity of amblyopia. Therefore the present screening program used in Sweden seems adequate. Children with low visual acuity in one or both eyes are detected and referred at the screening age of 4 . At any age squint or any other eye disease observed by parents or at the health centre are referred directly to an ophthalmologist for examination and treatment.

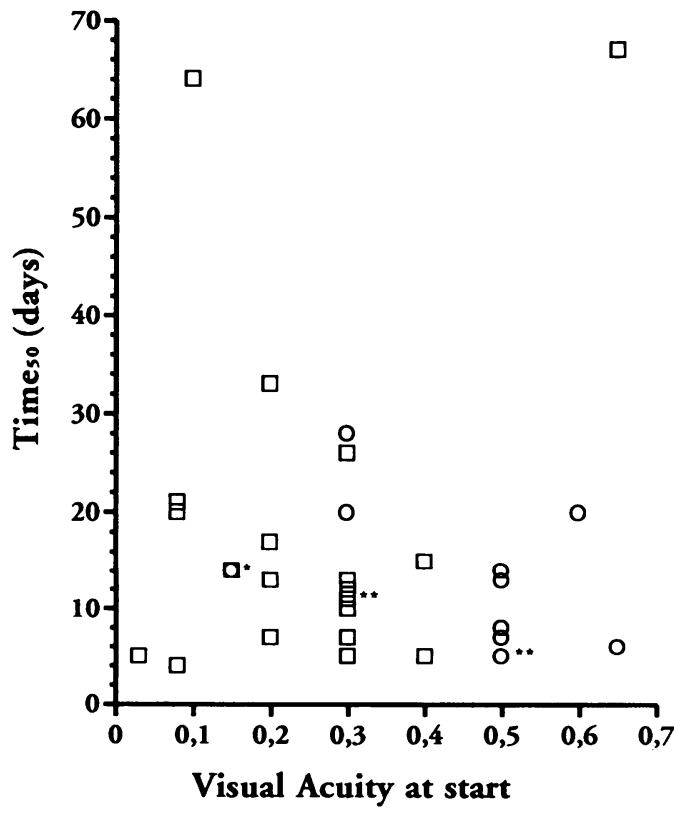

Figure 4B

Figure 4 Initial rate of improvement. Time (days) to halfpoint $\frac{(\log \text { VA start }+\log \text { VA cure) }}{2}$ between VA at start and 
Table 2 Clinical characteristics of patients $(n=8)$ with low compliance

\begin{tabular}{|c|c|c|c|c|}
\hline \multirow[t]{2}{*}{ Case no } & \multirow[t]{2}{*}{ Age } & \multicolumn{2}{|c|}{ Visual acuity } & \multirow{2}{*}{$\begin{array}{l}\text { Time to cure } \\
\text { (days) }\end{array}$} \\
\hline & & Start & Cure & \\
\hline \multicolumn{5}{|l|}{ Strabismus } \\
\hline 18 & 2 & 0.3 & 0.5 & 156 \\
\hline 38 & 2 & $<0.1$ & 0.5 & 206 \\
\hline & 9 & 0.1 & $0 \cdot 2^{\star}$ & $150^{\star}$ \\
\hline \multicolumn{5}{|l|}{ Anisometropia } \\
\hline 11 & 4 & 0.5 & 0.8 & 228 \\
\hline 39 & 4 & $0 \cdot 3$ & $0.6^{\star}$ & $>1000^{\star}$ \\
\hline 42 & 7 & $0 \cdot 2$ & $0.6^{\star}$ & $325^{\star}$ \\
\hline 43 & 6 & $0 \cdot 2$ & $0 \cdot 6^{\star}$ & $130^{\star}$ \\
\hline 44 & 4 & 0.2 & 0.8 & 412 \\
\hline
\end{tabular}

^Cure not achieved; no co-operation obtained for further treatment.

Another major finding in our study was that good compliance to any form of amblyopic treatment was associated with more than $95 \%$ cure, whereas only $50 \%$ of the children with low compliance were cured. Neumann $e t a l^{5}$ found that a higher proportion of younger children $(2-5 \cdot 5$ years) were more compliant to patching than children older than 8 years (72\% versus $47 \%$ ). They concluded that this was probably the primary reason for the higher percentage of treatment failures in older children. Our study shows that low compliance to any form of therapy is also the primary factor for treatment failure in children at any age between 2 and 7 years. The children with low compliance in our study were on average about one year older at start of therapy.

Compliance thus seems to be the main factor for cure, except for those rare cases with therapyresistant amblyopia, in which amblyopia is probably induced within the first years of life. ${ }^{614}$ Emphasis should therefore be placed on adherence to therapy during amblyopia treatment, and initial and repeated information about the importance of compliance to treatment should be given to the family. Follow-up even after a week or two weeks of treatment is rewarding for the visual acuity has improved in most cases (Fig 5), and the child and the parents are hereby encouraged to go on with the treatment. One possible reason for the low improvement rate of severe amblyopia found in some studies ${ }^{8}$ could be low compliance.

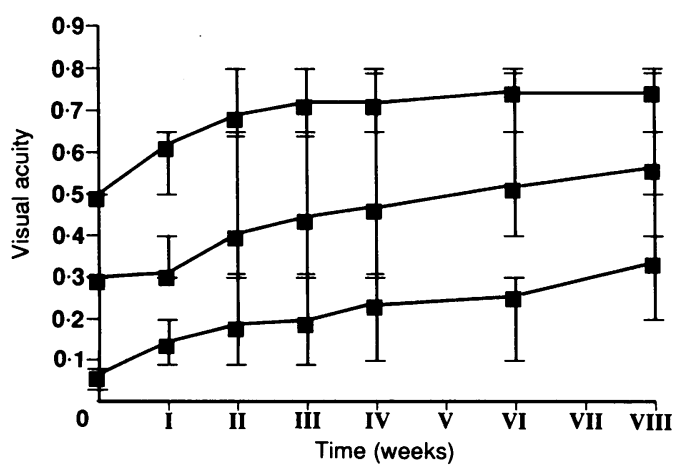

Figure 5 Rate of improvement during the first weeks of amblyopia treatment in relation to visual acuity of the amblyopic eye at start. The range of VA at one to eight weeks after start is presented for three representative groups of cured compliant children with initial VA of $\leqslant 0 \cdot 1(n=5), 0 \cdot 3$ $(n=10)$, and $0 \cdot 5(n=5)$. The ranges overlap for the two groups, with higher initial VA between four and eight weeks as indicated and for the group with initial $V A \leqslant 0 \cdot 1$ at eight weeks. Identical range limits are indicated by double horizontal bars.
Although we put much time and effort into information we had eight out of 44 with low or no compliance. Five of these children had moderate amblyopia - that is, visual acuity 0.1 to 0.3 due to anisometropia without strabismus. In the anisometropic group with mild amblyopia - that is, visual acuity above 0.4 - we had no problems with compliance. Neumann $e t a l^{15}$ also reported the lowest rate of compliance in the group with anisometropic amblyopia.

The question arises why it is so difficult for the anisometropic child with visual acuity of $0 \cdot 1-0 \cdot 3$ to accept a patch. A probable answer is that lower degree of motivation for treatment was present for patients in this group with straight eyes. Our five anisometropic children with low compliance were treated for long periods (years), and periods of adequate compliance were obtained. All of them had improvement of at least three lines and two were finally cured, so their amblyopia is probably not resistant to therapy. However, our study cannot rule out the possibility that the therapeutic responses in anisometropia and strabismic amblyopia are different. It is interesting to note that the two types of amblyopia have differences in psychophysical characteristics. ${ }^{16-18}$

The treatment time needed to achieve cure, namely one line difference or less between the eyes, was highly dependent on compliance but not on the initial type or degree of amblyopia. All but two of the compliant children were cured within three months of treatment regardless of initial visual acuity or type of amblyopia in contrast to the children with lower compliance. Treatment regimen and type of amblyopia therefore seems to have limited impact on the time needed to achieve cure. Similarly, Oliver $e t$ $a l^{5}$ and Neumann $e t a l^{15}$ reported that most of the improvement of visual acuity occurred during the first three months of treatment independently of type or initial degree of amblyopia. The rate of initial improvement in our compliant children showed no obvious correlation with visual acuity at the start of treatment, though a tendency may be observed. Neumann et $a l^{15}$ have reported a quicker recovery of visual acuity after three months of treatment in children (aged 2-7 years) with more severe amblyopia at the start.

Our study hypothesis that age is a strong factor in the outcome of amblyopia treatment was not supported. Instead we found that the age of the child was not critical for a successful result. However, the number of children above 5 years of age in our study was limited. Age was only weakly associated with the time needed to achieve cure in the age group 2-7 years in cases with good compliance. Similarly, Oliver and Nawratzki found that up to the age of 6 years good therapeutic results (about $65 \%$ ) were obtained in all age groups. They stated that most children with amblyopia can be effectively detected at the age of 4 years and concluded that this age probably is the best age for screening, also taking into account that the prevalence of amblyopia is lower below 3 years of age. ${ }^{4}$ At 2 years of age our strabismic children were cured somewhat quicker than the older ones, but this difference in cure rate is of limited practical importance if we consider the lower accuracy of acuity testing under screening conditions at earlier ages. Fulton 
and Mayer $^{19}$ reported similar results in a retrospective study of the relationship between age and duration of full-time occlusion in squinting children and demonstrated that older children show a slower rate of acuity improvement during patching. Sattler ${ }^{20}$ found a quick recovery within the first three months of treatment for 3-year-old children with squint than for those aged 7 or above.

Flynn and Cassady ${ }^{21}$ demonstrated a decreasing percentage of successful results and prolonged duration of therapy in amblyopic children with start of therapy after 5 years of age. Screening after the age of 6 is therefore probably suboptimal from the therapeutic point of view.

In summary, in our study, designed to be optimal for amblyopia treatment, we found that the patient's compliance was the main factor in achieving cure in most cases with strabismus and anisometropia in children between 2 and 7 years. Most of the improvement occurred during the first months and the majority $(>90 \%)$ were cured within three months irrespective of age between 2 and 7 years. Visual acuity screening at 4 years of age seems to be advantageous and efficacious in order to detect and thereafter initiate successful treatment of most cases of amblyopia without obvious squint.

This work was funded by a grant from the Swedish Medical Research Council (no. 02226), First of May Flower Annual Campaign for Children, and Handlanden Svenssons fond

We thank Maths Abrahamsson for help with the illustrations and for useful discussions and Lena Kjellberg for skilful assistance.

1 Nordlöw W, Joachimsson S. A screening test for visual acuity in four-year-old children. Acta Ophthalmol (Kbh) 1962; 40: 453-62.

2 Köhler L, Stigmar G. Vision screening of four-year-old children. Acta Paediatr Scand 1973; 62: 17-27.

3 Köhler L, Stigmar G. Visual disorders in 7-year-old children with and without previous vision screening. Acta Paediatr Scand 1978; 67: 373-7.

4 Oliver M, Nawratzki I. Screening of pre-school children for ocular anomalies. II. Amblyopia. Prevalence and therapeutic results at different ages. Br f Ophthalmol 1971; 55: 467-71.

5 Oliver M, Neumann R, Chaimovitch Y, Gotesman N, Shimshoni $M$. Compliance and results of treatment for amblyopia in children more than 8 years old. Am $\mathcal{J}$ Ophthalmol 1986; 102: 340-5.

6 von Noorden GK. Amblyopia: a multidisciplinary approach. Invest Ophthalmol Vis Sci 1985; 26: 1704-15.

7 Stewart-Brown SL, Haslum MN, Howlett B. Preschool vision screening: a service in need of rationalisation. Arch Dis Child 1988; 63: 356-9.

8 Ingram RM. Review of children referred from the school vision screening programme in Kettering during 1976-8. Br Med F 1989; 298: 935-6.

9 Ingram RM, Walker C, Billingham B, Lucas J, Dally S. Factors relating to visual acuity in children who have been treated for convergent squint. Br f Ophthalmol 1990; 74: 823 .

10 Lithander J. Kolt-Test Prüfung der Sehschärfe bei 2jährigen. Z Prakt Augenheilkd 1984; 5: 258

11 Hedin A, Nyman KG, Derouet B. A modified letter matching chart for testing young children's visual acuity. $\mathcal{F}$ Pediatr Ophthalmol Strabismus 1980; 17: 114-8.

12 Haase W, Hohmann A. Ein neuer Test (C Test) zur quantitativen Prüfung der Trennschwierigkeiten (crowding) Ergebnisse bei Amblyopi und Ametropi.Klin Monatsbl Augenheilkd 1988; 180: 210-5.

13 Cavazos H, Schulz E, Rassow B, Weseman W. Vergleich des Kindersehschärfetests nach Lithander (Kolt-Test) mit dem standardisiertem Landoltring. Klin Monatsbl Augenheilkd in standardi

14 Parks MM. Visual results in aphakic children. Am $\mathcal{f}$ Ophthalmol 1982; 94: 441-9.

15 Neumann R, Oliver M, Gottesmann N, Shimshoni M. Prognosis for occlusive therapy for strabismic and anisometropic amblyopia and for different initial depths of amblyopia. Chibret Int f Ophthalmol 1989; 6: 22-7.

16 Levi DM, Klein S. Differences in vernier discriminations for gratings between strabismic and anisometropic amblyopes. Invest Ophthalmol Vis Sci 1982; 23: 398-407.

17 Hess RF, Campbell FW, Zimmern R. Differences in the neural basis of human amblyopias: the effect of mean neural basis of human amblyopias: the
luminance. Vision Res 1980; 20: 295-305.

18 Sjöstrand J. Contrast sensitivity in children with strabismic and anisometropic amblyopia. A study of the effect of treatment. Acta Ophthalmol (Kbh) 1981; 59: 25-33

19 Fulton AB, Mayer DL. Esotropic children with amblyopia: effects of patching on acuity. Graefes Arch Clin Exp Ophthalmol 1988; 226: 309-12.

20 Sattler $\mathrm{CH}$. Erfahrungen über die Beseitigung der Amblyopie und die Wiederherstellung des binokularen Sehakts bei Schieldenden. Z Augenheilkd 1927; 63: 19-37.

21 Flynn JT. Cassady JC. Current trends in amblyopia therapy. Ophthalmology 1978; 85: 428-50. 